\title{
Assessing Pre-Service Teachers’ Soccer Offensive Game Performance Ability
}

\author{
Weiyun Chen ${ }^{*}$, Kristin Hendricks \\ University of Michigan School of Kinesiology, Ann Arbor, USA \\ Email: ${ }^{*}$ chenwy@umich.edu
}

Received April 19 ${ }^{\text {th }}$, 2012; revised May $17^{\text {th }}$, 2012; accepted June $2^{\text {nd }}, 2012$

\begin{abstract}
This study aimed to design and validate the Soccer Offensive Game Performance Instrument (SOGPI) that assessed pre-service teachers' offensive game abilities in soccer. One investigator videotaped two 10 -minute 3 vs. 3 soccer games played by twelve pre-service teachers at the end of a soccer unit. Two investigators independently coded 480 offensive game behaviors with the SOGPI. The results of $t$-tests indicated that the SOGPI was a valid instrument to differentiate the players' overall game performance and individual game components between the novice and the experience groups. The results of the inter-rater reliability indicated that the SOGPI was a reliable instrument to assess pre-service teachers' offensive game abilities in soccer.
\end{abstract}

Keywords: Game Performance; Game Behaviors; Game Assessment

\section{Introduction}

Improving and assessing pre-service teachers' game performance competency is a challenging task in physical education teacher education programs (McCullick, 2001; Siedentop, 2002). This is because pre-service teachers will be future physical education teachers and key agents for implementing quality physical education programs in schools. The Beginning Physical Education Teacher Standards (National Association for Sports and Physical Education (NASPE), 2009) address the growing consensus that pre-service teachers' skill-based movement performance competency in a variety of sports and physical activities is the "central core of our content area" (Siedentop, 2002: p. 373). Pre-service teachers' game performance competency is one of the important factors contributing to effective physical education teaching (McCullick, 2001; NASPE, 2009). Pre-service teachers' game performance competency directly links to their ability to demonstrate correct techniques of a skill and to guide students applying the skills with tactical purposes. Effective demonstration helps students see what proper forms of a skill look like and elicits students to imitate the key features of a skill. The pre-service teachers' deep understanding of tactical concepts underlying the skill application helps them design progressively sequential learning tasks and gradually apply game-like tasks for students. Adequate game performance competency prevents the pre-service teachers from falling into teaching an introductory unit cycle, which will eventually constrain K-12 students from meeting the content standards (McCullick, 2001, NASPE, 2009; Siedentop, 2002). Designing a reliable and valid assessment instrument for assessing pre-service teachers' game performance competency in the context of a specific sport meets the pragmatic need for helping pre-service teachers learn and demonstrate competent game performance levels. To date, there is no assessment instrument to serve for this specific purpose. Therefore, the aim of this study was to design and validate the

${ }^{*}$ Corresponding author.
Soccer Offensive Game Performance Instrument (SOGPI). This study designed the SOGPI as a both formative and summative assessment tool to assess how well the pre-service teachers demonstrated offensive game performance competency in soccer during small-sided soccer games.

\section{Theoretical Framework}

\section{A Situated Learning}

A theoretical framework for this study was the situated learning perspective. Lave and Wenger (1991) argue that learning take places in the interactive context of learners, activity, and environment. Learning normally occurs when learners socially interact with one another and when they actively engage in an authentic activity in a culturally-embedded environment. The first key principle of the situated learning perspective is that knowledge construction occurs in a real world situation. Situated learning usually involves application of knowledge and engagement of tasks in a contextualized learning domain. It emphasizes that people should present knowledge and tasks in rich authentic application situations rather than in abstract and de-contextualized settings. Learners need to engage in authentic learning tasks that involve application of related concepts and skills to solve a learning problem (Kirk \& MacPhail, 2002; Lave \& Wenger, 1991; Rovegno, 2006).

The second key principle of the situated learning perspective is that learning involves social interaction and collaboration (Lave \& Wenger, 1991). Learners socially interact with other participants in the "community of practice" to which each learner brings his/her knowledge, experiences, beliefs, and behaviors. Learners share their questions, confusions, ideas, understanding, and cultural norms with one another as they engage in learning activities. The social interaction shapes learners' beliefs, cognitive thinking, and social behaviors (Kirk \& MacPhail, 2002; Lave \& Wenger, 1991; Rovegno, 2006).

Situated learning goes beyond the social interaction and 
authentic learning. It emphasizes the fact that learning involves learners' active engagement with other learners, socially organized tasks, and a culturally situated environment. Learners bring their prior knowledge, experiences, and perceptions to actively engage in learning tasks within the physically, socially, and culturally constituted learning environment. As learners interact with the learning task, they actively make decisions about what and how they are going to cognitively, physically, and socially respond to the demands, nature, and changing forms of a learning task. Learners are active thinkers, decision makers, and action enactors (Kirk \& MacPhail, 2002; Lave \& Wenger, 1991; Rovegno, 2006).

\section{A Situated Learning Perspective of Developing Game Competency}

From a situated learning perspective, the dynamic interaction of tactical awareness and skill execution in contextual game situations results in an individual's game performance competency (Alexander \& Penney, 2005; Gréhaigne, Wallian, \& Godbout, 2005; Kirk \& MacPhail, 2002; Mitchell, Oslin, \& Griffin, 2006; Rovegno, 2006). Tactical awareness is an individual's ability to make appropriate decisions about what to do with skills in order to solve tactical problems in dynamic game situations (Mitchell et al., 2006). Skill execution is an individual's ability to execute on-the-ball skills and off-the-ball movements that are tactically appropriate for a particular game situation. Skill execution requires individuals to demonstrate how to perform the skills and movements in a specific game situation in order to successfully accomplish tactical decisions. Tactical awareness and technical skills are intertwined and interdependent cornerstones essential for successful game performance. The more effectively players can integrate these two components into game situations, the more likely they can demonstrate competent game performance and become skillful game players (Gréhaigne et al., 2005; Mitchell et al., 2006). An individual's demonstration of game performance competency only occurs in authentic game contexts. To obtain an actual profile of an individual's game performance competency, there is a need for using sound assessment instruments to assess individuals' game performance behaviors related to tactical awareness and skill execution in authentic game situations (Alexander \& Penney, 2005; Gréhaigne et al., 2005; Memmert \& Harvey, 2008).

\section{A Situated Learning Perspective of Game Performance Assessment}

A situated learning perspective places an emphasis on engaging in authentic tasks which parallel to real world settings (Kirk \& MacPhail, 2002; Lave \& Wenger, 1991; Rovegno, 2006). According to the principle of the situated learning perspective, the essential dimensions of a game performance assessment should capture the key game components for players to solve tactical and technical problems in situated game settings (Kirk \& MacPhail, 2002; Rovegno, 2006). Building on previous studies, Oslin, Mitchell, and Griffin (1998) designed the Game Performance Assessment Instrument (GPAI) to assess an individual player's abilities to make decisions, move appropriately, and execute skills across invasion, net, field, and target games. Oslin et al. (1998) stressed that seven game components, including Adjust, Base, Cover, Decision Made, Guard/Mark, Skill Execution, and Support, were essential for successful game per- formance, but not all of them were applicable to a specific game form. For example, all components except "base” were applicable to invasion games such as soccer and basketball. On the other hand, all components except "guard/mark" were essential for successful field games like baseball and softball. Similarly, all components except "guard/mark" and "support" were applicable to net games such as volleyball and tennis. The GPAI was a flexible observation instrument for teachers and researchers to choose to observe any game components related to a particular game form for specific instructional purposes.

The situated learning perspective requires a definition of each component in the game performance assessment to be game specific (Kirk \& MacPhail, 2002; Rovegno et al., 2001). This is because the game is context specific even though two games belong to the same invasion game form. For example, soccer and basketball games are in the same invasion game form. Although they share similar tactical concepts such as moving to open space to create a passing lane, soccer or basketball has its own specific game rules, unique game court dimensions, a given number of players, and specialized skills (Oslin et al., 1998; Richard, Godbout, \& Gréhaigne, 2000). These essential characteristics make each game context different from another. Therefore, some technical and tactical concepts are game specific and situated (Gréhaigne, Godbout, \& Bouthier, 1997). For example, in basketball, a player may set a screen for his/her teammates to create open space for his/her teammates. However, in soccer, a player can not use pick and roll tactics to create open space for his/her teammates because the soccer game rules do not allow a player to block his/her teammate's opponent's pathways. In addition, creating open space has specific meaning to each invasion game because the dimensions of a game field and the size of a playing area are different from one another. For instance, in soccer, a right midfield player and a center midfield player may use an overlap run to create open space, but a right midfield player and a left midfield player rarely use an overlap run to switch playing areas because of the large size of the soccer field. However, due to a limited court area in basketball, a right forward and a left forward frequently relocate to opposite sides of the playing area to create open space. The playing area, movements, and positions of teammates and opponents all influence how a player creates open space to receive the ball (Launder, 2001). Changing and specific game contexts constantly shape how a player makes tactical and technical decisions, executes skills, and provides support for teammates. Different ways of handling the ball, moving to open space on the available playing area, and interacting with teammates and opponents make game contexts different and specific.

\section{Needs for Elaborating on the GPAI}

Due to the fact that each invasion game is context specific within the similar tactical concepts, the global feature of the GPAI may not always provide a valid and reliable assessment of game performance for various invasion games (Nadeau, Richard, \& Godbout, 2007; Richard et al., 2000). Using the GPAI to measure individual players' game performance behaviors in soccer, basketball, and volleyball games, Oslin et al. (1998) reported that in $66 \%$ of the cases, the GPAI game components could differentiate players' observable game behaviors and abilities. These components included Skill Execution, Decision Making, Support, Game Performance, and Game Involvement. The results indicated that the construct validity of the GPAI was moderate, 
which might be the result of a broad definition of each game component, observational coding protocols, and calculation of the coding with the GPAI (Memmert \& Harvey, 2008).

Assessing individual players' game performance behaviors in dynamic game situations is much more challenging than assessing students' motor skill performance in closed and predetermined conditions (Gréhaigne \& Godbout, 1998). Examining the major concerns of the GPAI in relation to the definitions and coding system, Memmert and Harvey (2008) noted that a broad definition of certain dimensions of the GPAI and subjective coding protocols made it very difficult for independent coders to judge if a player's specific game component was appropriate/efficient or inappropriate/inefficient, especially for offthe-ball movements.

To improve the objectivity and reliability of coding a specific game component, it is critical to make assessment criteria observable and specific to situated game contexts (Mitchell \& Oslin, 1999). It is imperative to narrowly define each game component and describe the coding protocols as specifically as possible (Memmert \& Harvey, 2008). Although scholars increasingly recognized the comprehensive and flexible advantages of the GPAI and researchers conducted studies on validation of the instrument in select invasion and net games, the current GPAI has its own limitations related to the definition of game components and subjective coding protocols (Memmert \& Harvey, 2008).

\section{Purpose of the Study}

There is a pragmatic need for modifying the global feature of the GPAI to make the game performance assessment criteria and coding protocols more adaptable to a particular game context. The purpose of this study was to create a valid and reliable instrument for evaluating pre-service teachers' offensive game performance competency in soccer by examining inter-rater reliability, internal consistency, and construct validity of the instrument.

\section{Designing the SOGPI}

Oslin et al.'s GPAI (1998) provided guiding principles for designing the Soccer Offensive Game Performance Instrument (SOGPI) that assessed the pre-service teachers' offensive game ability in soccer. Two main reasons for choosing soccer included: 1) Soccer is a core curriculum content for K-12 physical education programs; and 2) Soccer is a unique invasion game which is different from basketball and field hockey etc. in terms of field, players, and rules. The SOGPI consisted of three essential game dimensions related to offensive game situations: Skill Execution, Decision Making, and Supports (Griffin, Mitchell, \& Oslin, 1997). The overall purpose of designing the SOGPI was to ensure a valid and reliable game performance assessment in the context of soccer in both research and teaching settings. Two specific rationales for designing the SOGPI were to make the game component criteria specific to soccer offensive game situations and to make the assessment coding protocols accommodate soccer offensive game situations.

\section{Definition of Game Components in the SOGPI}

From the situated learning perspective, the three essential game components including Skill Execution, Decision Making, and Support are game specific and contextual. It is necessary to identify essential game components and define each game component specifically and contextually. In the SOGPI, the Skill Execution game component included three sub-game components reflecting three essential offensive skills in soccer: Dribbling, Passing, and Shooting; the Decision Making game component consisted of three sub-game components including the Tactical Purposes of Dribbling, of Passing, and of Shooting that applied to game situations; and the Support game component comprised of two sub-game components including Creating Open Space and Keeping Width and Depth. Table 1 displays the definition of each game component in the SOGPI.

In Table 1, within the Skill Execution, the specific and observed offensive game skill criteria focused not only on the technical and tactical process of dribbling, passing, and shooting, but also on the end product of the motor responses, such as dribbling with control, passing with accuracy, and shooting with accuracy. As Gréhaigne et al. (2005) noticed, the application of motor skills in game contexts involves a dynamic interaction between technical and tactical processes and products. To solve tactical problems in game situations, a player should apply a specific skill with a specific tactical aim in order to adapt to specific game situations. For example, when using the SOGPI to assess a player's skill execution of passing, observers were looking for whether the player passed the ball with accuracy and in what appropriate game situations the player did so. In other words, the SOGPI defined that when a teammate is open, has good supporting positions, or has the best shooting position, the player passing a ball with accuracy is appropriate for the game situations.

Table 1.

Definition of each game component and the rating scale in the SOGPI.

\begin{tabular}{ll}
\hline \multicolumn{1}{c}{ Game Components } & \multicolumn{1}{c}{ Definition of Each Sub-Game Component } \\
Skill Execution: & $\begin{array}{l}\text { 1. Dribbling: Dribbles a ball while changing pace and directions and maintaining control of the ball. } \\
\text { 2. Passing: Passes accurately when a teammate is open, has a good supporting position, or has the best shooting position. }\end{array}$ \\
3. Shooting: Shoots when appropriate and scores a goal. \\
1. Attempts to dribble to take on/beat defender, advance the ball, protect the ball, or adjust positions. \\
2. Attempts to pass to set up a shot, move the ball, or beat defender. \\
3. Attempts to shoot when appropriate.
\end{tabular}


The Decision Making component meant choosing what to do with the ball in response to a tactical problem (Mitchell et al., 2006). In this study, we defined a player's decision making ability as an attempt to use a specific skill in response to a specific tactical problem. For example, the SOGPI described a player's appropriate decision making for dribbling, passing, and shooting in specific tactical situations. Similarly, the SOGPI identified two specific game components in relation to the Support dimension (off-the-ball movements). They defined that a player without the ball used specific tactical movements to create open space and to create a passing lane to receive a ball in the context of a soccer game. In summary, the definition of each game component in the SOGPI described the tactical processes and technical products of dribbling, passing, and shooting, the tactical purposes of making a decision about these three offensive skills, and the tactical processes of the support (off-the-ball movements) in more specific and explicit ways.

\section{Rating Scales in the SOGPI}

The SOGPI used the event recording tally method to help evaluators objectively assess an individual player's specific offensive game behaviors. An evaluator observed and recorded the presence or absence of the specified game behaviors on each sub-game component with a tally mark when the observed players' team gained possession of the ball. On the SOGPI assessment sheet, each game component contained five columns marked 1 through 5, representing five segments of the team gaining possession of the ball. Five team possession segments were available to be observed and coded for each individual player's demonstration of the game components. Table 1 described the rating scale of the SOGPI. For instance: a "+” mark indicated that an individual player demonstrated the definition of each sub-game component within the corresponding column (parallel to appropriate/efficient response in the GPAI (Mitchell et al., 2006)); a “-” mark illustrated that an individual player did not demonstrate the definition of each sub-game component within the corresponding column (parallel to inappropriate/ inefficient response in the GPAI (Mitchell et al., 2006)); a “/” mark indicated that a specific sub-game component was not applicable to an individual player within the corresponding column.

\section{Coding Protocols of the SOGPI}

This study specified the coding protocols to help an evaluator objectively assessing an individual player's offensive game performance with the SOGPI. The coding protocols included: 1 ) observing the identified player's offensive game behaviors until the team scored a goal, the ball went out of bounds, or the other team intercepted the ball; 2) coding the player's offensive game performance of each game component using the event recording tally method; 3) re-watching the player's offensive game behaviors if necessary; 4) switching the observation and coding of the opponent's offensive game behaviors once the team lost possession of the ball; 5) taking turns observing and coding each pair of individual players' offensive game behavior until the match was over using the above procedures; 6) rewinding the DVD to the very beginning of the game for watching and coding a new pair of individual players' offensive game behaviors throughout the game until completing the coding of all players' offensive game performances. 15 hours are needed for an evaluator to learn and use the coding protocols to practice coding the taped game play with the SOGPI.

\section{Methods}

\section{Research Participants and Settings}

Twelve physical education teacher education (PETE) students (8 females and 4 males) who enrolled in one secondary methods course voluntarily participated in this study. They were junior and senior PETE majors with the average age of 21 years old with a 2.27 standard deviation of age. Eleven students were White and one Asian. Of the participants, one played varsity soccer in high school and three had previous soccer club playing experience, while eight participants never played on a soccer team before taking this course.

This methods course consisted of four units: soccer, basketball, volleyball, and team hand ball. In the soccer unit, the course instructor used the tactical games approach (Mitchell et al., 2006) to teach the PETE students five soccer lessons, each lesson lasting two hours in length. Throughout the five soccer lessons, the PETE students learned the techniques of passing, dribbling, and shooting and the tactical purposes of applying these skills within the context of game positions and tactical game situations, while also following game rules. They also learned: 1) how to use a give and go, overlap run, diagonal run, and checking run to create open spaces and passing lanes; and 2) how to maintain width and depth to support the ball carrier in a modified and situational game play setting.

\section{Data Collection}

\section{Videotaping the Game Plays}

At the end of the soccer unit, the instructor organized the students into four teams of three players based on their skill levels to make each team as reasonably balanced as possible. One research assistant videotaped the students' playing 10-minute 3 vs. 3 games on a regular basketball court in the gym. During the videotaping of the games, the research assistant placed the camcorder in an unobtrusive corner of the gymnasium, adjusted the camcorder's angles, and zoomed in and out to ensure that all six players' on-the-ball skills and off-the-ball movements were in view at all times.

\section{Coding the Taped Game Plays}

Prior to officially coding the two videotaped game play sessions, two investigators spent an estimated 20 hours practicing the observing and coding of two players' offensive game actions with the SOGPI until they were satisfied with the performance indicators of each game component, the rating scales, and the coding protocols. The first investigator was the course instructor who had used the tactical games approach to teach the pre-service teachers the secondary teaching methods course including the soccer unit for 13 years. The second investigator was the research assistant who earned a bachelor degree in physical education teacher education and took the secondary methods course with the primary investigator prior to involvement in this study. She played on a varsity soccer team in high school and coached a junior varsity soccer team for a year.

Next, the two investigators independently coded each player's offensive game behaviors with the SOGPI assessment sheet by strictly following the coding protocols. They independently coded the 12 participants' demonstration of a total of 480 offensive game 
behaviors when their teams were in possession of the ball during the 10-minute 3 vs. 3 soccer games.

\section{Interviewing the Participants}

To gather the participants' background information, the first investigator conducted one 20-minute formal interview with each participant at the end of the unit using semi-structured interview questions. The interview questions asked the information about the pre-service teachers' athletic and coaching background in soccer. The first investigator used the mini tape recorder to record the interview and the second investigator transcribed the audio-taped interviews.

\section{Data Analysis}

This study used the formula: \% $\mathrm{R}=$ numbers of agreement $\div$ (numbers of agreement + numbers of disagreement) $* 100$ (Van der Mars, 1989) to calculate the inter-rater reliability (IR) of the SOGPI. The two investigators independently coded each participant' playing a 10-minute 3 vs. 3 soccer game using the SOGPI assessment sheet with the two-point "yes" and "no" rating scale. In other words, the investigator marked "+” representing "yes" on the corresponding cell of the assessment sheet to indicate the participant demonstrating the definition of the sub-game component. In contrast, the investigator marked “-” representing "no" on the corresponding cell of the assessment sheet to indicate the participant not displaying the definition of the sub-game component (See Table 2). If the definition of the sub-game component was not applicable to the participant's movement, the investigator marked "/” on the corresponding cell of the assessment sheet. Out of a total of 480 coded game behavior responses, the two investigators initially worked together to check each investigator's coding results cell by cell marked with either “+”, “-”, or "/” by circling the different coding sign (result) on the two separate coding sheets. Then, they tallied the number of different coding signs (results) and the number of same coding signs (results), respectively. Last, they inserted the corresponding numbers into the correct places on the above formula to calculate the inter-rater reliability of the SOGPI. In addition, to examine the internal consistency of the SOGPI, this study used Cronbach's alpha correlation coefficient to analyze the data set of 480 coded game responses.

In this study, we calculated the index score of each essential game component through multiplying the percentage of efficient/appropriate game responses with the total number of times the player gained possession of the ball/ the total number of times the player performed the off-the-ball movement. For example,

1) Skill Execution Index (SEI) is: $\mathrm{SEI}=$ (the number of efficient game responses $\div$ (the number of efficient game responses + the number of inefficient game responses) $) \times$ the total number of the player's gaining possession of the ball.

2) Decision Making Index (DMI) is: $\mathrm{DMI}=$ (the number of appropriate game responses $\div$ (the number of appropriate game responses + the number of inappropriate game responses) $) \times$ the total number of the player's gaining possession of the ball.

3) The Support Index (SI) is SI = (the number of appropriate game responses $\div$ (the number of appropriate game responses + the number of inappropriate game responses) $) \times$ the total number of the player's off-the-ball movement.

4) The Overall Game Performance Index (OGPI) is OGPI = $(\mathrm{SEI}+\mathrm{DEI}+\mathrm{SI}) \div 3$ (Mitchell et al., 2006).
We used PASW statistics 18 to calculate the index score of each individual game component and the overall game performance for each participant. To determine the construct validity of the SOGPI, this study used the independent t-test to examine if the SOGPI could differentiate the players' offensive game performance ability between the experienced and inexperienced groups. This study used three essential game components and one overall game performance variables to measure the pre-service teachers' game performance ability in soccer.

\section{Results}

\section{Reliability of the SOGPI}

To assess the inter-rater reliability, the investigators checked the degree of coding consistency between the two independent coders. Among a total of 480 coded offensive game behavior responses, the number of agreement was 459 , while the number of disagreement was 21 . According to the formula

$($ IR $\%=459 \div(459+21))$, the inter-rater reliability of the

SOGPI was $96 \%$. The result indicated a high consistency between the two raters' judgment (van der Mars, 1989).

To examine the internal consistency of the SOGPI, the investigators used Cronbach's alpha reliability coefficient to analyze the data set of 480 coded offensive game behavior responses. The alpha reliability coefficient for the total scale of the SOGPI was .95. The alpha value of the total scale was higher than .70 which indicated that the SOGPI had a high degree of internal consistency reliability (Stevens, 2002).

\section{Construct Validity of the SOGPI}

Table 2 shows each individual player's Skill Execution Index (SEI), Decision Making Index (DMI), Support Index (SI), and Overall Game Performance Index (OGPI).

Seven participants (four females and three males) who did not have previous soccer playing experience prior to participating in the methods course were classified into the novice group. In contrast, five participants (three females and two males) who had several years of soccer playing experience in high school and club were classified into the experience group. Table 3 shows the descriptive statistics of the SEI, DMI, SI, and OGPI between the two groups.

To determine construct validity of the SOGPI, first, this study used the independent t-test (Welch's formula of t-test due

Table 2.

Descriptive statistics of each individual player's index score of five game components.

\begin{tabular}{cccccc}
\hline Groups & Individual & SEI & DMI & SI & OGPI \\
\hline \multirow{2}{*}{ (Novice) } & Nicole & 1.00 & 1.00 & 2.00 & 1.30 \\
& Megan & 1.00 & 2.00 & 4.00 & 2.30 \\
& Rachel & 4.00 & 5.00 & 3.00 & 4.00 \\
& Bryan & 3.00 & 5.00 & 4.00 & 4.00 \\
& Ellen & 3.00 & 4.00 & 5.00 & 4.00 \\
& Mike & 3.00 & 4.00 & 5.00 & 4.00 \\
& Beth & 2.00 & 4.00 & 7.00 & 4.30 \\
& Nick & 4.02 & 6.02 & 4.00 & 4.73 \\
& Amy & 5.00 & 5.00 & 5.00 & 5.00 \\
& Caroline & 6.00 & 7.00 & 5.00 & 6.00 \\
& Alex & 6.02 & 6.00 & 7.00 & 6.30 \\
& Ellice & 8.01 & 9.00 & 8.00 & 8.36 \\
\hline
\end{tabular}


Table 3.

Descriptive statistics of the coded offensive game behaviors between the two groups.

\begin{tabular}{ccccccccc}
\hline \multirow{2}{*}{ Groups } & \multicolumn{2}{c}{ SEI } & \multicolumn{2}{c}{ DMI } & \multicolumn{2}{c}{ SI } & \multicolumn{2}{c}{ OGPI } \\
\cline { 2 - 9 } & $M$ & $S D$ & $M$ & $S D$ & $M$ & $S D$ & $M$ & $S D$ \\
\hline 1 (Novice) & 2.43 & 1.13 & 3.57 & 1.51 & 4.28 & 1.60 & 3.41 & 1.15 \\
2 (Experience) & 5.81 & 1.48 & 6.60 & 1.15 & 5.8 & 1.64 & 6.08 & 1.44 \\
\hline
\end{tabular}

to the unequal sample size between groups) to examine the mean scores of the OGPI between the two groups. The OGPI provided a comprehensive view of a player's game performance competency (Mitchell et al., 2006). As seen in Table 3, the mean score of the OGPI in the novice group was 3.41, while the mean score of the OGPI in the experience group was6.08. The independent $t$-test revealed that the mean score of the OGPI in the novice group was significantly lower than that of the OGPI in the experience group $(t=-3.44, d f=7.5, p=0.1)$. The result of t-test indicated that the SOGPI was a valid instrument to distinguish the players' overall offensive game ability between the novice and the experience groups.

Next, this study used the independent $t$-test (Welch's formula of t-test) to examine if each individual essential game component differentiated the two groups. With regards to the SEI, the independent $t$-test indicated that the novice group $\left(\mathrm{M}_{\text {novice }}=2.43\right)$ scored significantly lower than the experience group $\left(\mathrm{M}_{\text {experience }}=5.81\right)(t=-4.29, d f=7.21, p<.01)$. Regarding the DMI, the $t$-test yielded a significant difference $(t=-3.44, d f=8.76, p<.01)$ between the novice group $\left(M_{\text {novice }}=3.57\right)$ and the experience group $\left(M_{\text {experience }}=6.60\right)$. With respect to the SI, the $t$-test indicated no significant difference $(t=-1.59, d f=8.6, p<.05)$ between the novice group $\left(\mathrm{M}_{\text {novice }}=4.28\right)$ and the experience group $\left(\mathrm{M}_{\text {experience }}=5.8\right)$. The results indicated that the SOGPI could differentiate the players' ability of Skill Execution and Decision Made between the novice group and the experiences group, but not for Support in this study.

\section{Discussion}

This study was central to examining the inter-rater reliability, internal consistency, and construct validity of the SOGPI. The inter-rater reliability of the SOGPI was $96 \%$ and the alpha reliability coefficient of the SOGPI total scale was .88. The results indicated a high and stable consistency between the two coders who independently coded a total of 480 game behavior occurrences and a high degree of internal consistency of the SOGPI. The findings might be associated with the specific definition of each sub-game component, the event record coding procedures, and the coding protocols.

This study used the situated learning perspective as the guiding principles to define each sub-game component in the SOGPI specific to authentic game situations. First, to design a reliable assessment for assessing an individual player's offensive game ability in soccer, the SOGPI adopted Oslin et al.'s (1998) three offensive game components essential to invasion games including Skill Execution, Decision Making, and Support (off-the-ball movements). The constructs of the SOGPI reflected the extent to which a player demonstrated offensive game behaviors in soccer. Second, the SOGPI determined and defined tactical and technical processes and products of the three essential skills within Skill Execution game component. It also identified and described three specific game components under Decision Making game component: attempting to dribble, pass, and shoot for specific tactical purposes relevant to specific game situations. Similarly, for Support game component, it identified and described how to create open space and maintain width and depth while attacking. Third, in line with Memmert and Harvey's (2008) call for a specific definition of a game component, the investigators narrowly and precisely defined the criteria of each sub-game component on the SOGPI. The articulated definition of each sub-game component of the SOGPI helped the observers have a better understanding of what specific game performance behaviors they were looking for as they were coding an individual player's game play.

The two-point rating scales of the SOGPI with specified coding protocols helped the observers objectively judge whether or not a player demonstrated the defined characteristics of each sub-game component. This also helped observers know what to focus on observing and when to code occurrence of a specific game behavior. In this study, while watching the videotaped game play, the observer only focused on observing one individual player's game behaviors at a time. When the team's possession of the ball changed, the observer stopped the DVD player and then coded whether or not the player demonstrated the criteria of each game component. In other words, the observer only focused on observing and coding what game behavior the individual player actually displayed throughout a possession. For example, in one segment of the team's possession of the ball, an observer may notice the player's exhibition of the following game behaviors. First, the player dribbles the ball while changing directions and speeds without losing the ball for taking on his/her opponent. Next, the player passes the ball accurately to his/her teammate when the teammate moves to the best shooting position for setting up a shot. Then, the player uses a give and go tactic to move to open space when his/her teammate dribbles to adjust positions and then shoots at the goal. Now, the observer stops the DVD and then codes the player's game behaviors as follows: 1 ) marks "+" in the first column of dribbling and passing and marks "/” in the first column of shooting within Skill Execution game component; 2) marks “+” in the first column for attempting to dribble and attempting to pass and marks "/” in the first column for attempting to shoot within Decision Making game component; and 3) marks "+" in the first column of actively creating open space and marks "/” in the first column of maintaining width and depth under Support game component. In short, the specified objective rating scales and the coding protocols helped the observers understand how to distinguish between specific game components and how to code a specific game performance behavior.

This study examined the construct validity of the SOGPI. The $t$-tests indicated that the SOGPI was a valid instrument to differentiate the players' overall game performance between the novice and experience groups. Furthermore, the results of the $t$-tests revealed that the players showed significant differences in SEI and DMI between the two groups. The results implied that the players in the experience group demonstrated more appropriate game behaviors in dribbling, passing, and shooting of the Skill Execution dimension than their counterparts. Likewise, players in the experience group were more likely to choose 
the skills to accommodate specific game situations. In contrast, the players in the novice group tended to choose the skills without tactical purposes. For example, the players in the experience group dribbled the ball to pass their defenders, outmaneuver their defenders, protect the ball, and/or adjust their positions. On the contrary, the players in the novice merely dribbled the ball without the intention of using dribbling as a means to solve tactical problems. Similar to the findings of this study, Oslin et al. (1998) reported the significant differences in the Skill Execution and the Decision Making between the high- and lowgame performance groups.

Contradictory to the results of Oslin et al. (1998), the results of the t-test in this study showed no significant difference in the Support game component between the novice and the experience groups. The results suggested that in this study the preservice teachers without the ball seemed to know when to move tactically to create a passing lane. Likewise, they seemed to know when to spread out strategically to provide adequate support for the ball carrier due to their taking the secondary methods course, in which the instructor used the tactical games approach to teaching them how to move tactically as described above in the methods section.

This study suggests that the SOGPI is a theoretically sound and psychometrically supported measure for assessing pre-service teachers' soccer offensive game performance competency. This study used the situated learning perspective as the theoretical framework to design the SOGPI. Based on the key principle of authenticity in the situated learning perspective, the definition of each sub-game component in the SOGPI reflected the authentic nature of the game performance. To ensure a reliable and valid assessment of an individual player's offensive game ability in the "real world" gymnasium setting with the SOGPI, this study specified the objective rating scales and the coding protocols. With the three key attributes, the SOGPI was able to help the observers know 1) what game performance behaviors they should focus on observing; 2) how to distinguish between specific game components; and 3) how to code a specific game performance response. The most significant contribution of the SOGPI is to help future researchers and teacher educators objectively assess pre-service teachers' soccer offensive game ability while watching a videotaped game play.

The definition of each sub-game component and the rating scales of the SOGPI with specified coding protocols also make peer assessment in real physical education classes possible and feasible. This study suggests that pre-service teachers may use the SOGPI as a peer assessment tool to assess their peers who are playing the small-sided soccer games. The peer assessment is one of the authentic assessment tools (Lund \& Kirk, 2002). It is an effective teaching strategy to involve students in the learning and teaching process. To extend this study, researchers may examine students' perspectives of and experiences in using the SOGPI to assess their peers' game performance during game play. Researchers may further examine the validity and reliability of the SOGPI using broad samples of pre-service teachers in various PETE programs within the cross-sectional and/or longitudinal research designs.

\section{REFERENCES}

Alexander, K., \& Penney, D. (2005). Teaching under the influence: Feeding games for understanding into the sport education develop- ment-refinement cycle. Physical Education and Sport Pedagogy, 10, 287-301. doi:10.1080/17408980500340901

Carmines, E. G. \& Zeller, R. A. (1991). Reliability and validity assessment. Newbury Park: Sage Publications.

Gardner, H. (1992). Assessment in context: The alternative to standardized testing. In B. R. Gifford, \& M. C. O’Connor (Eds.), Changing assessment: Alternative view of aptitude, achievement and instruction (pp. 77-119). Boston: Kluwer Academic.

Gréhaigne, J., \& Godbout P. (1998). Formative assessment in team sports in a tactical approach context. Journal of Physical Education, Recreation, and Dance, 69, 46-51.

Gréhaigne, J. F., Godbout, P., \& Bouthier D. (1997). Performance assessment in team sports. Journal of Teaching in Physical Education, 16, 500-516.

Gréhaigne, J. F., Walllian, N., \& Godbout, P. (2005). Tactical-decision learning model and students' practices. Physical Education and Sport Pedagogy, 10, 255-269.

Griffin, L. L., Mitchell, S. A., \& Oslin, J. L. (1997). Teaching sport concepts and skills: A tactical games approach. Champaign, IL: Human Kinetics.

Hastie, P. A. (1996). Student role involvement during a unit of sport education. Journal of Teaching in Physical Education, 16, 88-103.

Kirk, D., \& MacPhail, A. (2002). Teaching games for understanding and situated learning: Rethinking the Bunker-Thorpe Model. Journal of Teaching in Physical Education, 21, 177-192.

Lave, J., \& Wenger, E. (1991). Situated learning: Legitimate peripheral participation. New York: Cambridge University Press. doi:10.1017/CBO9780511815355

Launder, A. G. (2001). Play practice: The games approach to teaching and coaching sports. Champaign, IL: Human Kinetics.

Lund, J. L., \& Kirk, M. F. (2002). Performance-based assessment for middle and high school physical education. Champaign, IL: Human Kinetics.

McCullick, B. A. (2001). Practitioners' perspectives on values, knowledge, and skills needed by PETE participants. Journal of Teaching in Physical Education, 21, 35-56.

Memmert, D., \& Harvey, S. (2008). The game performance assessment instrument (GPAI): Some concerns and solutions for further development. Journal of Teaching in Physical Education, 27, 220-240.

Mitchell, S. A., \& Oslin, J. L. (1999). An investigation of tactical transfer in net games. European Journal of Physical Education, 4, 162-172. doi:10.1080/1740898990040205

Mitchell, S. A., Oslin, J. L., \& Griffin, L. L. (2006). Teaching sport concepts and skills: A tactical games approach $\left(2^{\text {nd }}\right.$ ed.). Champaign, IL: Human Kinetics.

Nadeau, L., Richard, J. F., Godbout, P. (2007). The validity and reliability of a performance assessment procedure in ice hockey. Physical Education and Sport Pedagogy, 13, 65-83. doi:10.1080/17408980701444718

National Association for Sport and Physical Education (NASPE) (2009). National standards \& guidelines for physical education teacher education (3rd ed.). Champaign, IL: Human Kinetics.

Oslin, J. L., Mitchell, S. A., \& Griffin, L. L. (1998). The game performance assessment instrument (GPAI): Development and preliminary validation. Journal of Teaching in Physical Education, 17, 231-243.

Richard, J. F., Gobout, P., \& Gréhaigne, J. F. (2000). Students’ precision and reliability of performance assessment in team sports. Research Quarterly for Exercise and Sport, 71, 85-91.

Rovegno, I. (2006). Situated perspectives on learning. In D. Kirk, D. Macdonald, \& M. O'Sullivan (Eds.), The handbook of physical education (pp. 262-264). London: Sage Publications.

Siedentop, D. (2002). Content knowledge for physical education. Journal of Teaching in Physical Education, 21, 368-377.

Stevens, J. (2002). Applied multivariate statistics for the social sciences $\left(4^{\text {th }}\right.$ ed.). Hillsdale, NJ: Lawrence Erlbaum.

Van der Mars, H. (1989). Observer reliability: Issues and procedures. In P. Darst, D. Zakrajsek, \& V. Mancini (Eds.), Analyzing physical education and sport instruction ( $2^{\text {nd }}$ ed., pp. 53-80). Champaign, IL: Human Kinetics. 